\title{
Umweltbewusstsein im Wandel \\ Trends und Tendenzen im Umweltbewusstsein
}

\author{
Seit den Anfängen der Umweltbewusstseins- \\ studien hat sich die deutsche Gesellschaft ge- \\ wandelt - und davon ist das Umweltbewusstsein \\ nicht unbeeinflusst geblieben. Im Folgenden \\ werden einige Thesen formuliert, die sich aus \\ den Umweltbewusstseinsstudien ableiten lassen \\ und die aktuelle soziologische Forschungen \\ zum Alltagsbewusstsein und zu soziokulturellen \\ Entwicklungen als Interpretationsrahmen einbe- \\ ziehen. Von Michael Schipperges, Maike Gossen, \\ Brigitte Holzhauer und Gerd Scholl
}

D ie Auswirkungen von politischer und wirtschaftlicher Globalisierung, Liberalisierung und Deregulierung ebenso wie die Ausbreitung neuer Informations- und Kommunikationstechnologien haben die Alltagswirklichkeit vieler Menschen verändert. Damit sind neue Chancen und Wahlmöglichkeiten, aber auch neue Herausforderungen und Probleme verbunden. Trotz eines andauernden Wirtschaftswachstums sind Ängste vor dem sozialen Abstieg und Sorgen um den Erhalt des gewohnten Lebensstandards bis weit in den gesellschaftlichen Mainstream vorgedrungen (Rheingold-Institut 2010; Bertelsmann Stiftung 2012; BMAS 2013; Deutscher Paritätischer Wohlfahrtsverband Gesamtverband 2014; OECD 2013; OECD 2014; IfD Allensbach/Axel Springer Verlag 2015; Piketty 2015).

Vor diesem Hintergrund ist auch die seit den 1960ern zu beobachtende Hinwendung zu postmateriellen Werten um die Jahrtausendwende einer neuen „postmodernen“ Werte-Synthese gewichen, in der materielle und postmaterielle, an Stabilität und Verlässlichkeit orientierte Werte mit für Veränderung und Innovation offenen Orientierungen koexistieren (Gensicke 2002; Klages/Gensicke 2004; Inglehardt/Welzel 2005; Kuckartz/Rheingans-Heintze 2006).

Auffällig ist ein bemerkenswertes Spannungsverhältnis bei vielen Menschen zwischen einer eher pragmatisch-kurzfristig ausgerichteten Bereitschaft zu Höchstleistung, Flexibilität und Mobilität sowie eher unterschwellig-langfristig wirksamen Sehnsüchten nach Verankerung, Geborgenheit und Entschleunigung. Vor diesem Hintergrund stellt sich die Frage: Welche Auswirkungen ergeben sich daraus für das Umweltbewusstsein (Schipperges et al. 2016)?

\section{Es ist ein Generationswechsel zu verzeichnen}

Das Umweltbewusstsein, wie es seit den 1960er Jahren in Deutschland entstanden ist, wurde im Wesentlichen zunächst von der damals jungen „Wertewandel-Generation“ getragen, für die postmaterielle Orientierungen wie Selbstverwirklichung, Emanzipation, Partizipation und eben auch Umweltschutz fest im individuellen Wertesystem verankert waren. Inzwischen ist eine neue Generation [1] herangewachsen, für die ein „postmoderner“ Werte-Mix charakteristisch ist: Pragmatismus, Flexibilität, Adaptivität und (Krisen-)Resilienz sind hierbei wichtige Merkmale, aber auch eine gewisse Sehnsucht nach Verlässlichkeit und Zukunftssicherheit (Schipperges 2010; Schipperges 2011; Hurrelmann/Albrecht 2014; Shell Deutschland Holding 2015).

In Bezug auf die ökologische Problematik verhalten sich die jüngeren Generationen taktischer und situativer - aber auch widersprüchlicher. So zeigt etwa die Studie „Umweltbewusstsein und Umweltverhalten junger Menschen“ (Gossen et al. 2015) einerseits, dass sich im Umweltbewusstsein der jungen Generation eine globale Perspektive auf die ökologische Problematik und darin eine besondere Bedeutung des Klimawandels feststellen lassen. Andererseits sind auf der Verhaltensebene traditionell als ökologisch-korrekt verstandene Handlungsmuster weniger wichtig geworden. Jedoch sind eine hohe Affinität zu Sharing und die Präferenz für niedrigschwellige und internetgestützte Formen des Umweltengagements zu beobachten. Je größer der Anteil dieser neuen Generation an der Gesamtbevölkerung wird, desto mehr werden sich Umweltbewusstsein und Umweltverhalten gegenüber den bislang bekannten Formen verändern.

\section{Der thematische Horizont hat sich verbreitert}

Das Umweltbewusstsein ist zunehmend globaler, generationenübergreifender und inhaltlich vielschichtiger geworden. Dies gilt in besonderem Maße für die jüngeren Generationen, aber auch insgesamt in der Gesellschaft. Die Umweltprobleme der sich entwickelnden Länder, die Lebenschancen der kommenden Generationen, die globale Klimaveränderung, der weltweite Zugang beispielsweise zu Land, Nahrung, Energie und Trinkwasser, das Verschwinden der Tropenwälder, aber auch zunehmende soziale Ungleichheit überall auf der Welt, werden deutlicher wahrgenommen. Alle Komponenten der 


\author{
„Die Umweltpolitik \\ muss die veränderten \\ Sichtweisen auf die \\ ökologische Problematik \\ und die daraus \\ resultierenden Umgangs- \\ weisen aufgreifen."
}

blematik heute in einem anderen Kontext gesehen wird: Erstens ist das Thema inzwischen weniger emotional aufgeladen; zweitens sind in der faktischen Umweltsituation hierzulande reale Fortschritte $\mathrm{zu}$ verzeichnen; und drittens ist für breite Bevölkerungsgruppen ein wirtschaftlicher Problemdruck entstanden, der mitunter die Umweltproblematik in den Hintergrund drängt. Die neu hinzugekommenen globalen Umweltprobleme (weltweite Klimaveränderung, Ressourcenverbrauch und Emissionen der sich entwickelnden Länder, Verschmutzung der Weltmeere) sind im heimischen Alltag dagegen weniger unmittelbar erfahrbar und entfalten daher einen geringeren Handlungsdruck.

\section{Nachhaltige Verhaltensweisen treten mit der Alltagsbewältigung häufiger in Konflikt}

Nachhaltigkeit, ökologische, ökonomische und soziale, werden mehr und mehr als miteinander verschränkt betrachtet. Der Kontext der Ökologiethematik wird inzwischen als vieldimensional und überaus kompliziert wahrgenommen und berührt grundlegende Fragen danach, was gutes Leben bedeuten kann. Dabei werden nicht nur die zu lösenden Probleme, sondern auch die Chancen, die eine konsequente Umwelt- und Nachhaltigkeitspolitik beinhaltet, deutlicher gesehen.

Bezüglich der Wahrnehmung eigener Gestaltungsmöglichkeiten und Verantwortungsübernahme ergibt sich allerdings ein ambivalentes Bild: So besteht einerseits der Anspruch, selbst aktiv auf Veränderungen hinzuwirken; andererseits ist aber auch die Delegation dieser Verantwortung an die Experten und qua Profession Zuständigen zu beobachten. Angesichts des grundlegenden Eindrucks von Krisenhaftigkeit und Unvorhersehbarkeit gesellschaftlicher Entwicklungen (Beck 1986; Rosa 2005; Bauman 2008; Schulze 2011) wird der Glaube an die Gestaltbarkeit von Zukunft geringer - obwohl gleichzeitig der Wunsch nach verlässlicher Gestaltung und Planbarkeit zunimmt.

\section{Der im Alltag empfundene Handlungsdruck nimmt ab}

Die wahrgenommene Bedeutung von Umweltproblemen (gemessen als offen erfragtes „aktuelles politisches Problem“ in den Umweltbewusstseinsstudien 2000 bis 2014) unterliegt im Zeitverlauf starken Schwankungen. In der Rangfolge der Häufigkeit, mit der die Umweltproblematik jeweils im Vergleich zu anderen politischen Fragen genannt wird, zeigt sich, dass andere und neue Themen mehr in den Vordergrund getreten sind (wie zum Beispiel soziale Sicherung oder aktuell Migration), während bezüglich der ökologischen Problematik eine Gewöhnung und Entdramatisierung stattgefunden hat [2].

Seit Anfang der 2000er Jahre sind mehrere Tendenzen festzustellen, die dazu führen, dass der Stellenwert der Umweltpro-
Nicht nur in den Umweltbewusstseinsstudien, sondern auch in den Markt-Media-Studien der Verlage von Publikumszeitschriften werden mit großen Stichproben Einstellungen und Verhalten auch im Hinblick auf ökologische Produkte gemessen. Die Entwicklung der entsprechenden Einstellungen im Zeitverlauf ergibt auf dieser Grundlage ein recht klares Bild: Demnach ist die Bereitschaft zu ökologisch korrektem Konsum - zumindest wie sie seit den 1990er Jahren verstanden und in den Umfragen abgefragt wurde - seit zwei Jahrzehnten rückläufig. Die Zahl derjenigen, die bereit sind, „für umweltfreundliche Produkte mehr auszugeben“, hat sich in diesem Zeitraum von 38 auf 19 Prozent halbiert (TdW 1994-2012). Die Orientierung an Produkteigenschaften wie „umweltfreundlich“, „natürlich“, „ökologisch“ oder „biologisch“ im alltäglichen Konsumverhalten geht seit den 1990er Jahren kontinuierlich zurück.

Angesichts einer zunehmenden Präsenz von Öko-Produkten im Handel, wie etwa auch von Bio-Lebensmitteln bei Discountern, und der hohen sozialen Erwünschtheit von Nachhaltigkeitsnormen überrascht dieser Befund auf den ersten Blick. Als Ursachen für diesen Rückgang können zum einen Umorientierungen und veränderte Prioritätensetzungen im Konsumalltag angesehen werden, wie zum Beispiel Schnäppchenmentalität, hybrider Konsum oder verstärkte ConvenienceBedürfnisse. Zudem sehen sich viele in der Bevölkerung einem wachsenden materiellen, zeitlichen und psychischen Druck im Alltagsleben ausgesetzt, der nachhaltigem Konsum entgegensteht.

Die im Durchschnitt rückläufige Entwicklung darf aber nicht darüber hinwegtäuschen, dass in bestimmten Bevölkerungssegmenten nachhaltiger Konsum an Verbreitung und Selbstverständlichkeit gewonnen hat. Insgesamt sind fair gehandelte, regionale, umweltfreundliche Produkte $\mathrm{zu}$ einem Ausweis bewusster Lebensführung und zum sozialen Distinktionsmerkmal geworden. Auch im umweltbewussten Konsum drückt sich damit ein zunehmendes Auseinanderdriften der Gesellschaft aus. 


\section{Die Skepsis bezüglich eines kontinuierlichen Wirtschaftswachstums nimmt zu}

Es sind nicht nur die ökologischen planetarischen Grenzen, die Zweifel an der Tragfähigkeit eines andauernden Wirtschaftswachstums aufkommen lassen; auch die ökonomischen Krisen haben der Wachstumskritik in den letzten Jahren neue Argumente geliefert. Damit sind der Kontext und der Bezugsrahmen der Ökologiethematik wieder fundamentaler geworden. Zweifel, ob ein unverändertes Weiter-so unserer Produktions- und Konsumptionsweise auf Dauer möglich ist, sind zunehmend verbreitet.

Vor dem Hintergrund der als Common Sense akzeptierten Überzeugung, dass nur der in der Wachstumskonkurrenz Überlegene überhaupt Überlebenschancen hat, ruft dies aber massive Sorgen nicht nur um den Erhalt des gewohnten Wohlstands, sondern auch existenzieller Art hervor (Rosa 2005; Bauman 2008). Die Beobachtung, dass ein Zurückbleiben bei Wachstum und Wettbewerb tatsächlich das „Aus“, den „Absturz“, das Verstoßenwerden ins Milieu der „Loser“ bedeutet, stellt die Menschen vor ein Dilemma. Unter den Jüngeren, die ihre Biografie und damit ihre Bewährungsprobe noch vor sich haben, ist dieses Empfinden besonders stark ausgeprägt.

\section{Alternative Produktions- und Konsumformen faszinieren}

Obwohl nur von wenigen tatsächlich selbst praktiziert, haben viele von regionalen Tauschringen, Carsharing, Urban Gardening, Reparaturcafés oder Wohngenossenschaften gehört und können sich vorstellen, bei so etwas mitzumachen. Bemerkenswert ist dabei, dass hier nicht sozial-normativ geprägte Aussagen getroffen werden, sondern dass positive Veränderungsphantasien zum Tragen kommen.

Eine Teilnahme an sozialen Innovationen entspricht nicht nur ökologischen Überlegungen, sondern folgt auch Bedürfnissen nach Vernetzung, sozialen Kontakten, Austausch mit anderen, der Nutzung faszinierender neuer Techniken. Eine wichtige Rolle spielt auch die Perspektive, nicht mehr von Großorganisationen und -institutionen abhängig zu sein, sondern sich selbstbestimmten sozialen Praktiken zuwenden zu können.

\section{Das Umweltbewusstsein ist hybride}

Die Menschen sehen sich zunehmend Zielkonflikten, kognitiven Dissonanzen und Dilemmata ausgesetzt. Das Bewusstsein für die Bedeutung von langfristig ausgelegten Strategien zur Bewältigung globaler Herausforderungen koexistiert mit der Orientierung an kurzfristigen Zielen im persönlichen Leben. Die Vielzahl und Komplexität der abstrakten Problemla- gen macht Kompromisse im konkreten Alltag nötig. Das Praktizieren von ökologisch-korrekten Verhaltensweisen geht einher mit dem Festhalten an umweltbelastenden Gewohnheiten. Nicht eine in Wertorientierungen fest verankerte ökologische Entschiedenheit steht bei den meisten im Vordergrund, sondern eine individuelle Mixtur aus Pragmatismus, Hedonismus und Verantwortungsbereitschaft.

So führen positive Umwelteinstellungen nicht automatisch zu einer guten Ökobilanz. Gerade Bevölkerungssegmente, in denen positive Umwelteinstellungen verbreitet sind, verbrauchen oft überdurchschnittlich viele Ressourcen. In diesen sozial eher gehobenen Milieus sorgen ein großzügiges Wohnumfeld und ausgeprägte Mobilitätsbedürfnisse für einen vergleichsweise schweren ökologischen Rucksack (Kleinhückelkotten et al. 2016).

\section{Fazit}

Das Umweltbewusstsein in Deutschland befindet sich offensichtlich in einem tiefgreifenden Wandel, von dem sich vieles derzeit erst in Umrissen erkennen lässt. Für die Umweltpolitik heißt das, neue Herausforderungen, aber auch neue Chancen, die sich aus den veränderten Sichtweisen auf die ökologische Problematik und daraus resultierenden Umgangsweisen mit dieser ergeben, in wachsendem Maße aufzugreifen. Für die Forschung ergibt sich die Aufgabe, das Konstrukt „Umweltbewusstsein“ in den aktuellen Zusammenhängen besser zu verstehen und zeitgemäß zu definieren. Insbesondere sind die Wahrnehmungs- und Deutungsmuster der jüngeren Generationen verstärkt in den Blick zu nehmen.

\section{Anmerkungen}

[1] Zu Konzepten des Generationswechsels vgl. Parnes et al. 2008 und Mannheim 2009.

[2] BUMB/UBA 2015; ein bemerkenswerter „Ausreißer" findet sich im Jahr 2012: Die zu diesem Erhebungszeitpunkt ungewöhnlich häufige Nennung von Umweltthemen kann auf die Reaktorkatastrophe von Fukushima und die daraufhin eingeleitete Energiewende in Deutschland zurückgeführt werden.

\section{Literatur}

Bauman, Z. (2008): Flüchtige Zeiten. Leben in der Ungewissheit. Hamburg.

Beck, U. (1986): Risikogesellschaft. Auf dem Weg in eine andere Moderne. Frankfurt am Main.

Bertelsmann Stiftung (2012): Mittelschicht unter Druck? Gütersloh.

BMAS (2013): Lebenslagen in Deutschland. Der Vierte Armuts- und Reichtumsbericht der Bundesregierung. Berlin.

BMUB/UBA (2015): Umweltbewusstsein in Deutschland 2014. Ergebnisse einer repräsentativen Bevölkerungsumfrage. Berlin.

Deutscher Paritätischer Wohlfahrtsverband Gesamtverband e. V. (2014): Die zerklüftete Republik. Bericht zur regionalen Armutsentwicklung in Deutschland. Berlin.

Gensicke, T. (2002): Individualität und Sicherheit in neuer Synthese? Wertorientierungen und gesellschaftliche Aktivität. In: Deutsche Shell (Hrsg.): Jugend 2002. Zwischen pragmatischem Idealismus und robustem Materialismus. Frankfurt am Main: 139-211. 
Gossen, M./Holzhauer, B./Schipperges, M./Scholl, G. (2015): Umweltbewusstsein in Deutschland 2014. Vertiefungsstudie: Umweltbewusstsein und Umweltverhalten junger Menschen. UBA-Texte 77/2015, Dessau-Roßlau.

Hurrelmann, K./Albrecht E. (2014): Die heimlichen Revolutionäre: Wie die Generation $Y$ unsere Welt verändert. Weinheim.

IfD Allensbach/Axel Springer Verlag (2015): Frauen der Sandwich-Generation. Ergebnisse einer repräsentativen Umfrage. Im Internet unter: www.ifd-allensbach.de/fileadmin/IfD/sonstige_pdfs/BdF_Studie_ Sandwich.pdf (zuletzt 01.09. 2016).

Inglehart, R./Welzel, C. (2005): Modernization, Cultural Change and Democracy. New York.

Klages, H./Gensicke, T. (2004): Wertewandel und Big-Five-Dimensionen. In: Schumann, S. (Hrsg.): Persönlichkeit. Eine vergessene Größe der empirischen Sozialforschung. Wiesbaden: 279-200.

Kuckartz, U./Rheingans-Heintze, A. (2006): Trends im Umweltbewusstsein. Umweltgerechtigkeit, Lebensqualität und persönliches Engagement. Herausgegeben vom Umweltbundesamt. Wiesbaden.

Kleinhückelkotten, S./Neitzke, H. P./Moser, S. (2016): Repräsentative Erhebung von Pro-Kopf-Verbräuchen natürlicher Ressourcen in Deutschland. UBA-Texte 39/2016, Dessau-Roßlau.

Mannheim, K. (2009): Das Problem der Generationen. In: Barboza, A./ Lichtblau, K. (Hrsg.): Schriften zur Wirtschafts- und Kultursoziologie. Wiesbaden: 121-166.

OECD (2015): In It Together: Why Less Inequality Benefits All. Paris.

OECD (2013): OECD Income Distribution Database; zitiert nach Michael Förster, Interview mit der Deutschen Welle.

Parnes, O./Vedder, U./Willer, S. (2008): Das Konzept der Generation. Eine Wissenschafts- und Kulturgeschichte. Frankfurt am Main.

Piketty, T. (2014): Das Kapital im 21. Jahrhundert. München.

Rheingold-Institut (2010): Die Absturz-Panik der Generation Biedermeier. Köln.

Rosa, H. (2005): Beschleunigung. Die Veränderung der Zeitstrukturen in der Moderne. Frankfurt am Main.

Schipperges, M./Gossen, M./Holzhauer, B./Scholl, G. (2016): Umweltbewusstsein und Umweltverhalten in Deutschland 2014. Vertiefungsstudie: Trends und Tendenzen im Umweltbewusstsein. UBA-Texte 59/2016, Dessau-Roßlau.

Schipperges, M. (2011): Soziale Milieus und Generationswechsel in der Stadtgesellschaft. Impulsreferat auf dem Kongress zum 20-jährigen Bestehen des Wuppertal Instituts.
Schipperges, M. (2010): vhw-Milieus 2010. In: Forum Wohnen und Stadtentwicklung 6: 285-293.

Schulze, G. (2011): Krisen. Das Alarmdilemma. Frankfurt am Main. Shell Deutschland Holding (Hrsg.) (2015): Jugend 2015. Eine pragmatische Generation im Aufbruch. 17. Shell Jugendstudie. Frankfurt a. M.

Typologie der Wünsche Intermedia (TdW) (1994-2012); wechselnde Herausgeber, u. a. Burda GmbH, IMUK. Offenburg.

AUTOREN + KONTAKT

Michael Schipperges ist Gründer und Geschäftsführer von sociodimensions, Institute for Socio-cultural Research.

Sociodimensions, Friedrich-Ebert-Anlage 60, 69117 Heidelberg, Tel. +4962216510861

E-Mail: schipperges@sociodimensions.com

Maike Gossen arbeitet im Forschungsfeld Unternehmensführung und Konsum im Institut für ökologische Wirtschaftsforschung (IÖW) als wissenschaftliche Mitarbeiterin und ist Doktorandin an der TU Berlin.

IÖW-Geschäftsstelle, Potsdamer Straße 105, 10785 Berlin. Tel. +49 30 884594-42,

E-Mail: maike.gossen@ioew.de

Dr. Brigitte Holzhauer ist selbstständige Sozial- und Marktforscherin.

Holzhauerei, Uhlandstraße 20, 68167 Mannheim. Tel.+49621 1504876, E-Mail: info@holzhauerei.de

Dr. Gerd Scholl leitet das Forschungsfeld Unternehmensführung und Konsum im Institut für ökologische Wirtschaftsforschung (IÖW). IÖW-Geschäftsstelle, Potsdamer Straße 105, 10785 Berlin. Tel. +49 30 884594-20, E-Mail: gerd.scholl@ioew.de
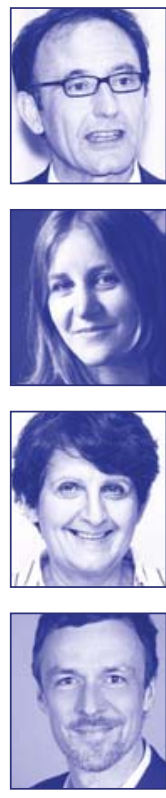

\section{Liebe Abonnent(inn)en, liebe Leser(innen)!}

Wir danken Ihnen für Ihre Treue und Ihr Interesse an unserer Zeitschrift Ökologisches Wirtschaften - und wir freuen uns darauf, Ihnen 2017 wieder spannende Schwerpunkte, Theorien und Konzepte vorstellen zu können!

Eine schöne Adventszeit und frohe Weihnachten wünschen

das Institut für ökologische Wirtschaftsforschung (IÖW), die Vereinigung für ökologische Wirtschaftsforschung (VÖW) und der oekom verlag
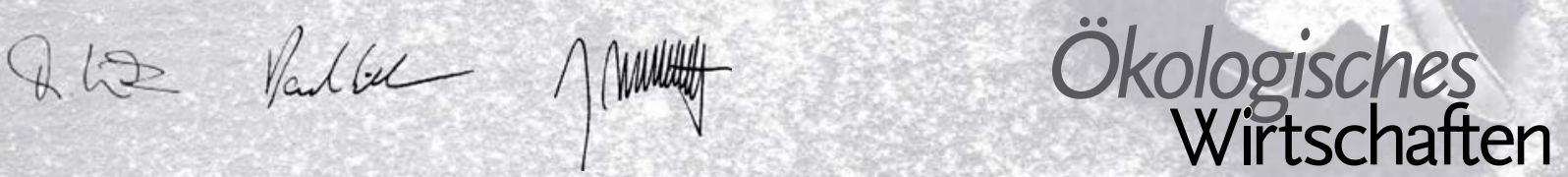